\title{
Indirect Genetic Characterization of Serratia
}

marcescens

\author{
A. Mohankumar (Corresponding Author) \\ Department of Zoology, Chikkanna Govt. Arts College, \\ Tirupur- 641 602, Tamilnadu, India \\ E-mail: moniver@satyam.net.in
}

R. Hari Krishna Raj

Department of Microbiology, Sengunthar Arts and Science College

Tiruchengode- 637 205, Namakkal Dt, India

E-mail: harikrishraaj@yahoo.co.in

Received: June 10, 2012 Accepted: June 24, 2012

doi:10.5296/jbls.v3i1.1935 URL: http://dx.doi.org/10.5296/jbls.v3i1.1935

\begin{abstract}
The pure culture isolate of Serratia marcescens, obtained from a clinical specimen was maintained in nutrient agar medium at $4^{\circ} \mathrm{C}$. It was genetically characterized by performing 16s rRNA sequence analysis, neighbour tree exposition, phylogenetic tree construction and peptide mass fingerprinting of serratiopeptidase, obtained from it. In order to obtain high purity of serratiopeptidase from the fermentation broth of pure culture isolate of Serratia marcescens, some separation and purification techniques were also explored. The results demonstrate that the combination of bio separation processes of ammonium sulfate precipitation with ion exchange chromatography is suitable for the separation and purification of serratiopeptidase from culture broth of Serratia marcescens. Peptide mass finger printing was performed and the amino acid sequence of serratiopeptidase was elucidated, compared and confirmed with clustal pair wise alignment database tool to confirm that the product produced and purified was serratiopeptidase from Serratia marcescens. Thus, indirectly Serratia marcescens has been completely genetically characterized using its therapeutically important product serratiopeptidase and the 16s rRNA sequencing of the bacteria.
\end{abstract}

Keywords: Genetic characterization, Serratia, Serratiopeptidase 


\section{Introduction}

Serratia marcescens was, for many years, believed to be non virulent but is now known to be an important opportunistic pathogen capable of causing a large variety of infectious diseases in humans (Lyerly and Kreger, 1979; Dorwart et al., 1975; Graber et al., 1965). In addition to the toxic lipo polysaccharides in its cell wall, the bacterium produces extracellular proteases. Serratia protease preparations of undemonstrated homogeneity have been reported to elicit skin, cornea and lung damage (Conroy et al., 1975). Various investigators have described procedures for obtaining purified preparation of Serratia proteases (Aiyappa and Harris, 1976; Lyerly and Kreger, 1979). Many of these isolates do not form pigment and are resistant to various antibiotics. The potential role of the protease produced by this organism was proteolytic destruction of corneal tissue and may be a cause of corneal lesion. A number of strains of Serratia marcescens have been reported to produce one to four proteases (Liu, 1961).

The present study is concerned with the indirect approach to genetically characterize Serratia marcescens by the production and purification of serratiopeptidase enzyme and its characterization apart from its 16s r RNA sequencing.

\section{Materials and Methods}

The strain of Serratia marcescens culture used in this study was isolated from a clinical specimen (corneal lesion).

\subsection{Genotypic characterization}

\subsubsection{DNA Extraction, PCR Amplification and Agarose Gel Electrophoresis}

From the overnight culture of pure culture isolate Serratia marcescens, the genomic DNA was extracted as per the procedure given by Helini Biomolecules, Chennai, India. Then the extracted DNA was amplified by the polymerase chain reaction. The amplified DNA was loaded into $2 \%$ agarose gel along with $10 \mu \mathrm{l}$ of $100 \mathrm{bp}$ DNA ladder. The band pattern was observed by viewing the gel in UV transilluminator.

\subsubsection{6s rRNA Sequence Analysis}

The PCR amplified product was subject to $16 \mathrm{~s}$ rRNA sequence analysis at Helini Biomolecules, Chennai, India using applied biosystems sequencer and an electropherogram data was obtained. The FASTA sequence obtained was compared with the database to confirm the identity of the Serratia species.

\subsubsection{Clustal Sequence Allignment for Neighbour and phylogenetic tree construction}

The clustal sequence alignment was done with the FASTA sequence obtained, comparing it with other species of Serratia sps. The clustal alignment tool helped in construction of a neighbour tree and phylogenetic tree to assign the isolated bacteria to a particular species in the taxonomical classification. Thus the sequence establishes the complete genetic identity of the organism. 


\subsubsection{Production and Purification of Serratiopeptidase}

The identity of the organism was also indirectly achieved with the help of the serratiopeptidase produced by it. Hence the isolated pure culture of Serratia marcescens, was inoculated in trypticase soy broth ( $\mathrm{pH} 7.2)$. Then the broth was incubated at $37^{\circ} \mathrm{C}$ for 20 to 24 hours. The supernatant fluids were obtained by centrifugation of the production medium at $10000 \mathrm{rpm}$ for 10 minutes and was used as the source of the enzyme.

\subsection{Dialysis}

Ammonium sulfate was added slowly to the culture supernatant fluids, with gentle stirring by using magnetic stirrer, to a final concentration of $60 \%$ saturation $(420 \mathrm{~g} / \mathrm{liter})$. After 24 hours, the precipitate was obtained by centrifugation. Then the precipitate was dissolved in $0.02 \mathrm{M}$ potassium phosphate buffer ( $\mathrm{pH} 7.0)$. This precipitant mixture was dialyzed overnight against phosphate buffer (pH7.0).

\subsection{Column Chromatography}

The dialysed sample was applied to a column of DEAE-Cellulose equilibrated with $10 \mathrm{mM}$ Tris Hydrochloride buffer (pH8.3). A linear gradient consisting of 2 liters of 10mM Tris HCL buffer (pH8.3) and 2 liters of the same buffer with $0.3 \mathrm{~m} \mathrm{NaCl}$ was then used.

Elute was collected for the measurement of absorbance at $280 \mathrm{~nm}$ and enzyme activity. The active fractions were pooled, dialyzed against double distilled water at $4^{\circ} \mathrm{C}$. The dialyzed sample was stored in refrigerator at $4^{\circ} \mathrm{C}$.

\subsection{SDS-PAGE}

Sodium dodecyl sufate polyacrylamide gel electrophoresis (SDS-PAGE) was performed to determine the molecular weight of the purified Serratiopeptidase. This purified serratiopeptidase was denatured by boiling for 2 mins in $2 \%$ SDS (Sodium dodecyl sulfate ) and $1 \%$ mercaptoethanol and then the denatured sample was loaded in the SDS-PAGE. Then the gel ( $30 \%$ acrylamide) was stained with $0.04 \%$ coomassie brilliant blue and it was destained with $7 \%$ acetic acid.

\subsection{Peptide Mass Finger Printing}

An artificial structure or an analogue design of the enzyme can be assigned and the serratiopeptidase identity can be confirmed only when the amino acid composition of the enzyme is known. Hence the enzyme was subject to peptide mapping by MALDI - TOF (Time Of Flight) - LCMS (Liquid Chromatography Mass Spectrophotometry) at TCGA research, New Delhi, India.

Pair Wise Alignment

The amino acid sequence was compared with the Serratiopeptidase in database to confirm and characterize the serratiopeptidase produced with the standard serratiopeptidase from Serratia marcescens. 


\section{Ml Macrothink}

Journal of Biology and Life Science

ISSN 2157-6076 2012, Vol. 3, No. 1

\section{Results}

\subsection{Agarose Gel Electrophoresis and PCR Amplification}

The isolated DNA was electrophoresed and demonstrated in plate: a and the PCR amplified DNA was electrophoresed and the PCR amplified DNA product is shown in plate:b .

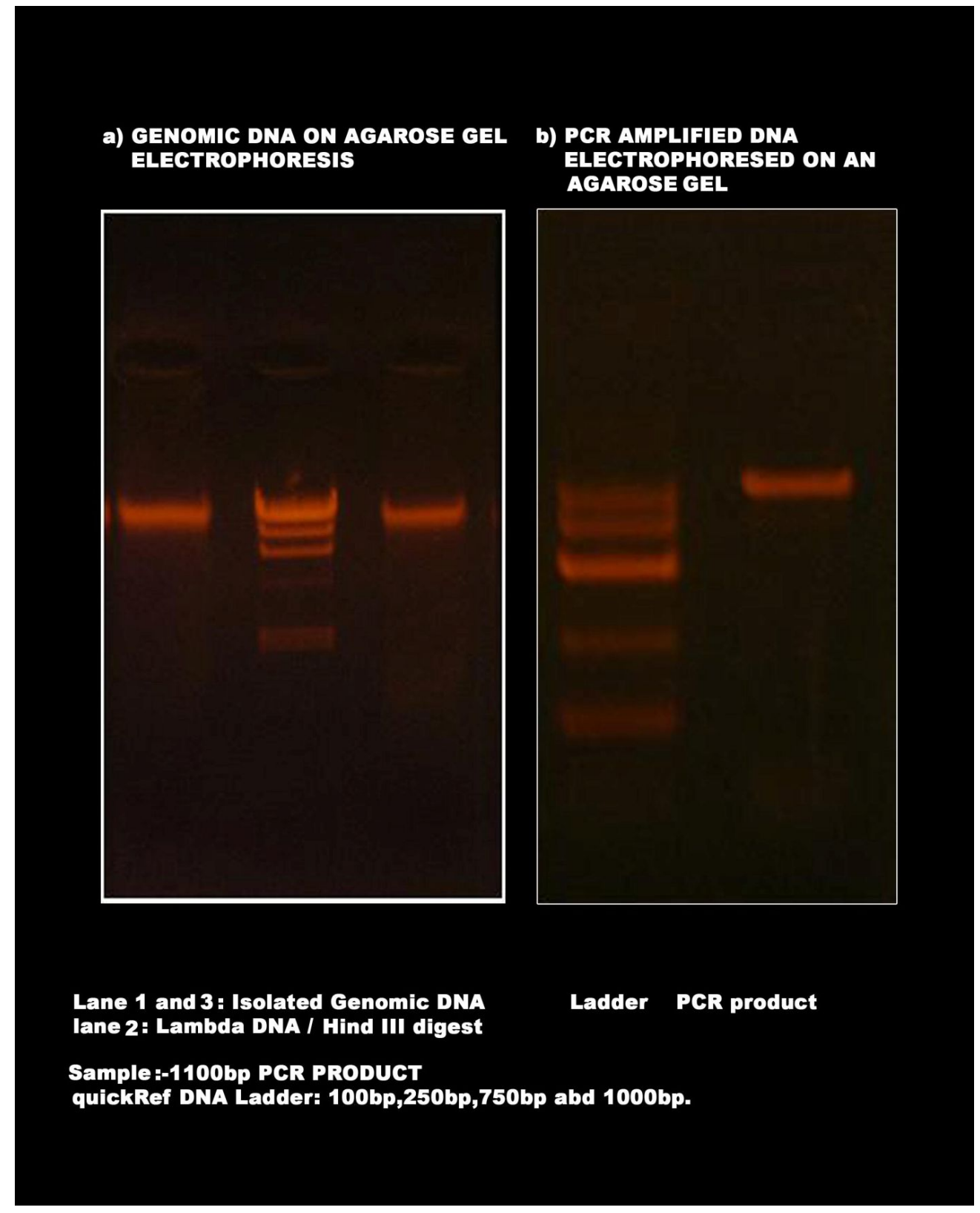

Plate $a \& b$

\subsection{6 s rRNA Sequence Analysis and phylogenetic tree}

The isolate (pure culture) obtained from the clinical specimen was subject to 16s rRNA sequence analysis. From the sequence analysis chromatogram (fig 1.0) the isolate was identified as Serratia marcescens. The sequence was compared with closely related sequences of other Serratia sps and a neighbour tree(fig. 2.0) was designed. The FASTA 
sequence (fig. 3.0) obtained was used for its gene submission in gene databank and structural analysis. Using the sequence obtained and the neighbour tree a "phylogeny tree" was constructed as shown in the figure4.0. Finally from this analysis, it was proved that the isolate was found to be a Serratia marcescens, confirming its identity.

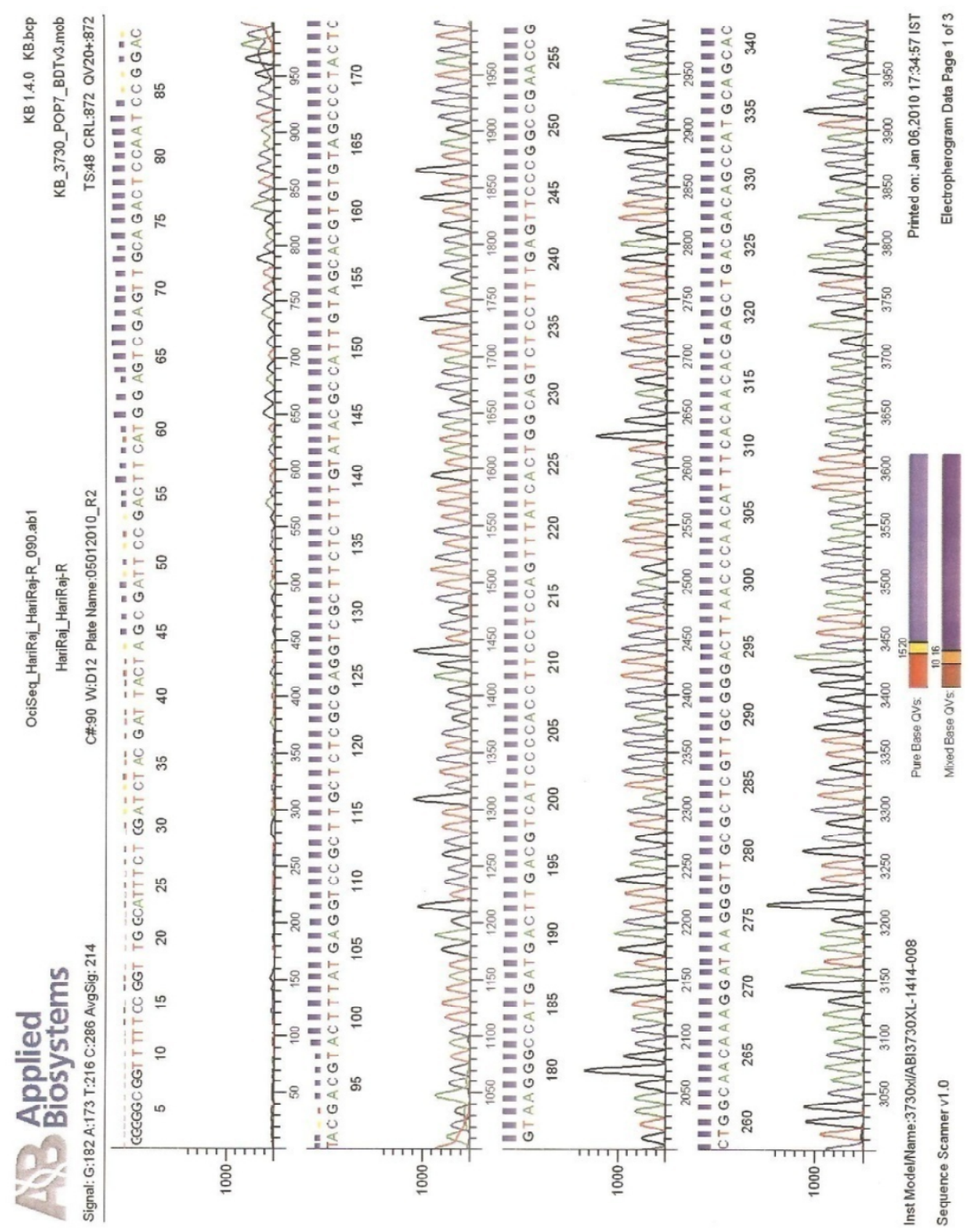




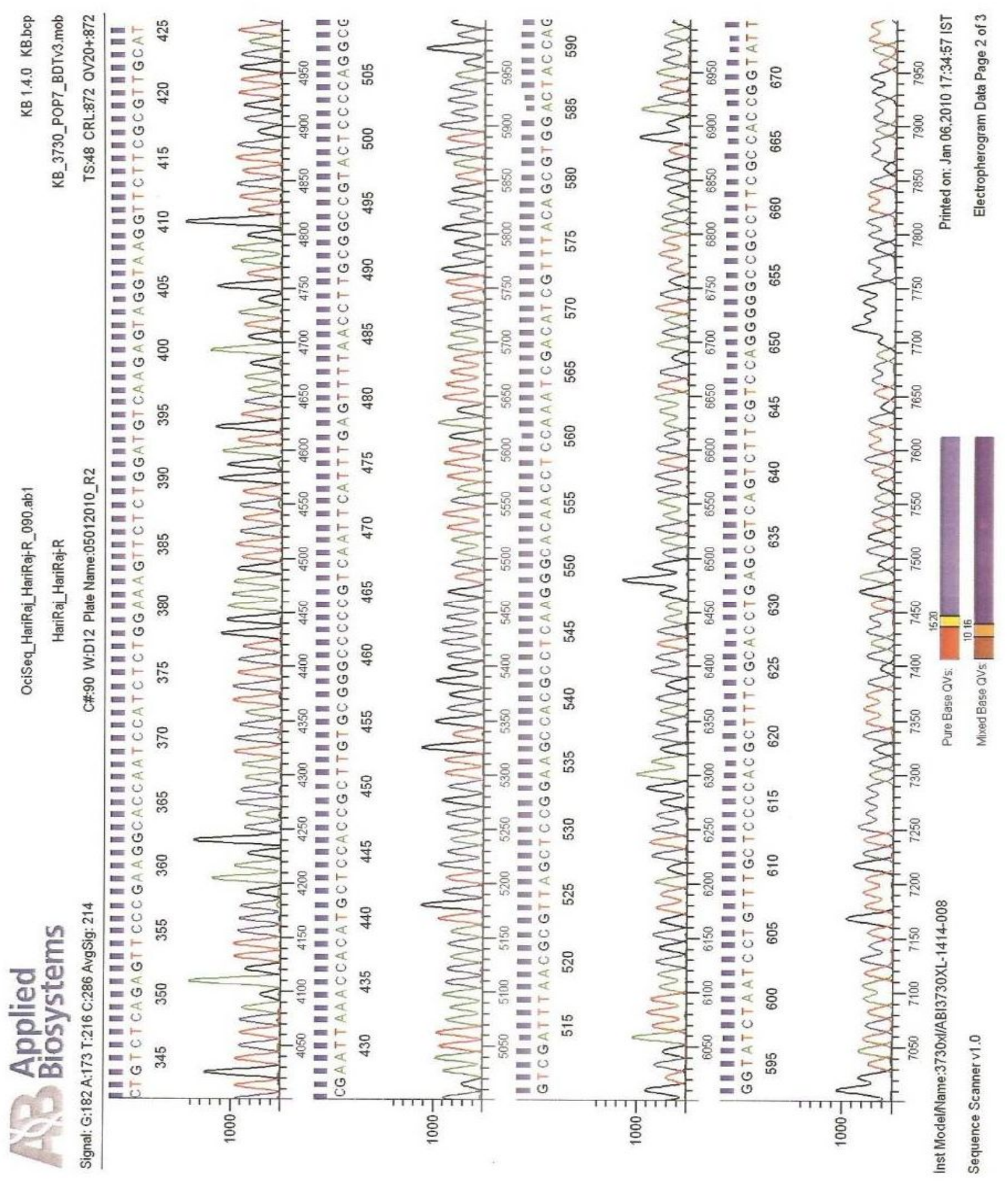




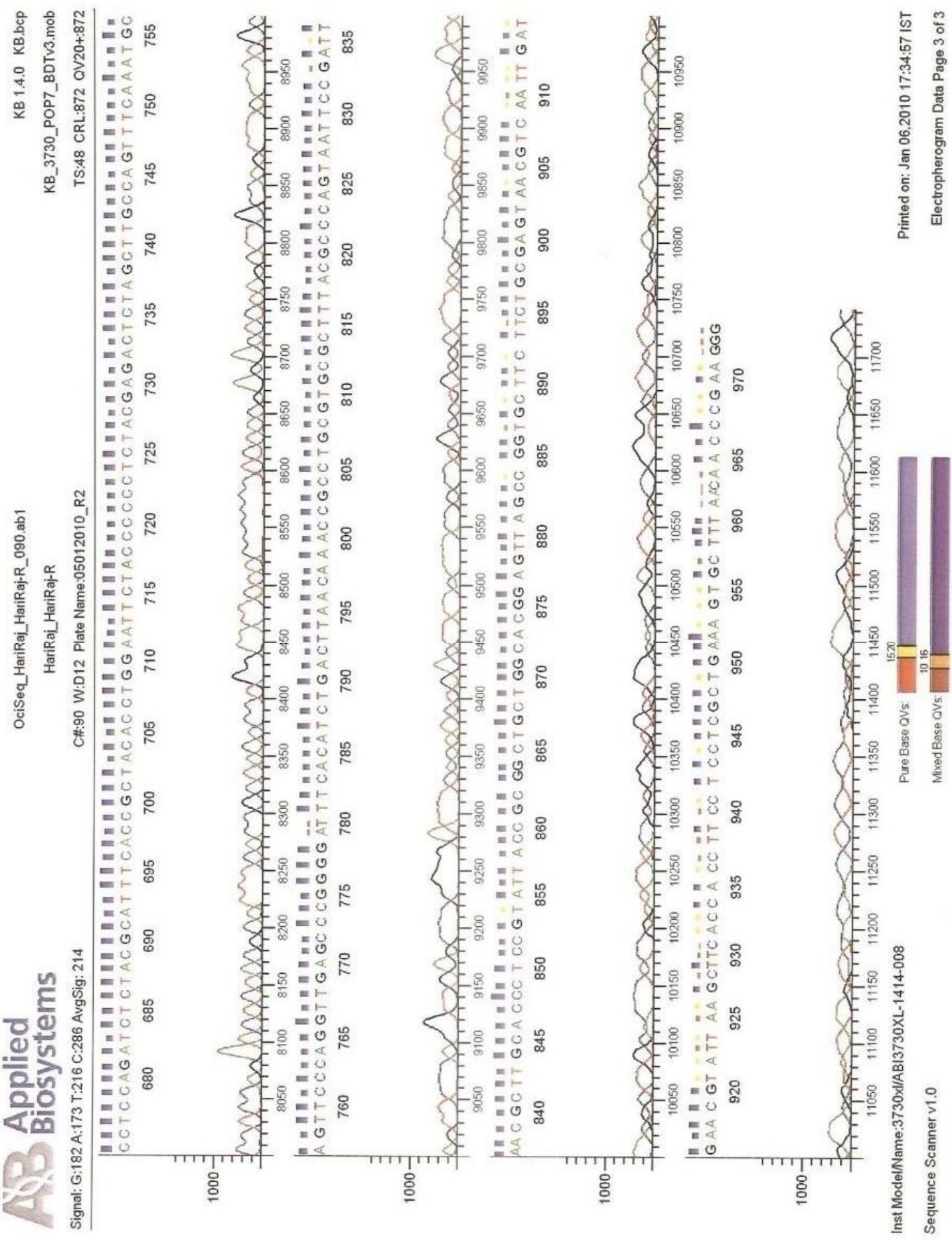

Figure 1. Chromatogram 
Populations

Neighbor-Joining/UPGMA method version 3.6a2.1

Neighbor-joining method

Negative branch lengths allowed

+Serratia nematodiphila

! +Serratia nematodiphila strain P36

! ! +Serratia marcescens

$2-3+-1$

! !+-4 +Serratia marcescens

! +-5 +Serratia marcescens strain CS265

! +Serratia marcescens strain MH6

+----------------------------------------------HariRaj

this is an unrooted tree!

Figure 2. Neighbour Tree

GGGGGCGGTTTTTCCGGTTGGCATTTCTGGATCTACGATTACTAGCGATTCCGAC TTCATGGAGTCGAGTTGCAGACTCCAATCCGGACTACGACGTACTTTATGAGGTC CGCTTGCTCTCGCGAGGTCGCTTCTCTTTGTATACGCCATTGTAGCACGTGTGTAG CCCTACTCGTAAGGGCCATGATGACTTGACGTCATCCCCACCTTCCTCCAGTTTAT CACTGGCAGTCTCCTTTGAGTTCCCGGCCGAACCGCTGGCAACAAAGGATAAGG GTTGCGCTCGTTGCGGGACTTAACCCAACATTTCACAACACGAGCTGACGACAGC CATGCAGCACCTGTCTCAGAGTTCCCGAAGGCACCAATCCATCTCTGGAAAGTTC TCTGGATGTCAAGAGTAGGTAAGGTTCTTCGCGTTGCATCGAATTAAACCACATG CTCCACCGCTTGTGCGGGCCCCCGTCAATTCATTTGAGTTTTAACCTTGCGGCCGT ACTCCCCAGGCGGTCGATTTAACGCGTTAGCTCCGGAAGCCACGCCTCAAGGGC ACAACCTCCAAATCGACATCGTTTACAGCGTGGACTACCAGGGTATCTAATCCTG TTTGCTCCCCACGCTTTCGCACCTGAGCGTCAGTCTTCGTCCAGGGGGCCGCCTTC GCCACCGGTATTCCTCCAGATCTCTACGCATTTCACCGCTACACCTGGAATTCTA CCCCCCTCTACGAGACTCTAGCTTGCCAGTTTCAAATGCAGTTCCCAGGTTGAGC CCGGGGATTTCACATCTGACTTAACAAACCGCCTGCGTGCGCTTTACGCCCAGTA ATTCCGATTAACGCTTGCACCCTCCGTATTACCGCGGCTGCTGGCACGGAGTTAG CCGGTGCTTCTTCTGCGAGTAACGTCAATTGATGAACGTATTAAGCTTCACCACC TTCCTCCTCGCTGAAAGTGCTTTAACAACCCGAAGGG

Figure 3. FASTA Sequence 


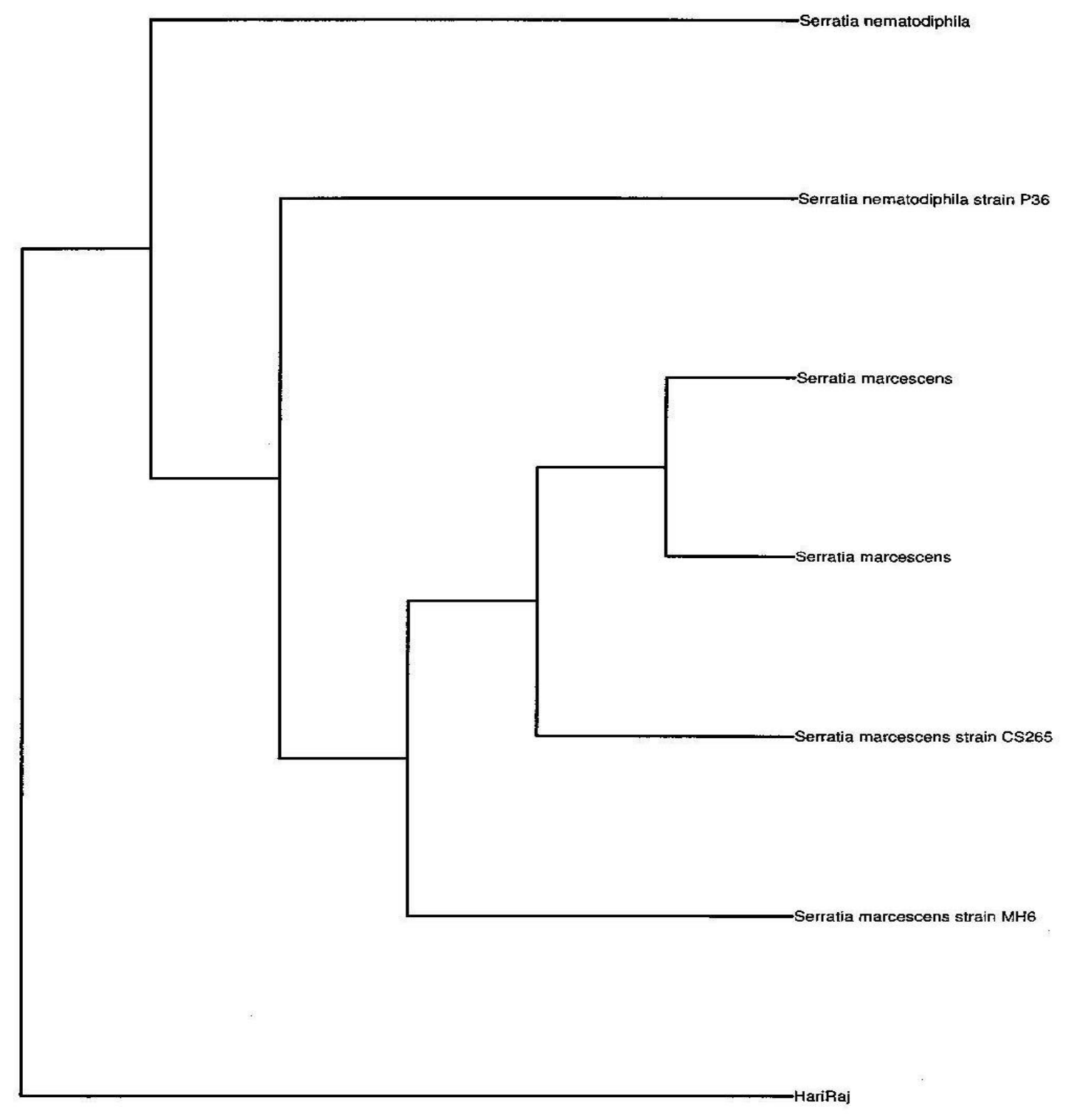

Figure 4. Phylogeny Tree

\subsection{Production and Purification of Serratiopeptidase Enzyme}

The isolated organism ie,.Serratia marcescens was inoculated in trypticase production medium for the production of serratiopeptidase enzyme. Then the fermentation broth was centrifuged at 10000rpm for 10-15 mins. After centrifugation, the pellet was discarded and the supernatant broth was stored in refrigerator for further use. The enzyme present in the supernatant broth was concentrated by performing ammonium sulfate precipitation. For the purification, the concentrated enzyme was dialyzed against phosphate buffer for the removal of impurities. Then for further purification the dialyzed enzyme was allowed to run in the DEAE cellulose pack. The purified sample was allowed to check the purification effect by performing SDS-PAGE Analysis. 


\section{Macrothink $\Lambda$ Institute"m}

\subsection{Characterization of the Serratiopeptidase Enzyme}

The purified enzyme solution was characterized by SDS-PAGE analysis. In this analysis, the molecular weight of the purified enzyme sample was found to $51 \mathrm{KDa}$, when compared to that of the standard protein marker (Plate: C). From the results of SDS-PAGE analysis it was confirmed that "Serratia marcescens" produced the purified serratiopeptidase and also it was to examine the effect of purification, and the results indicated that the final product obtained is homogenous.

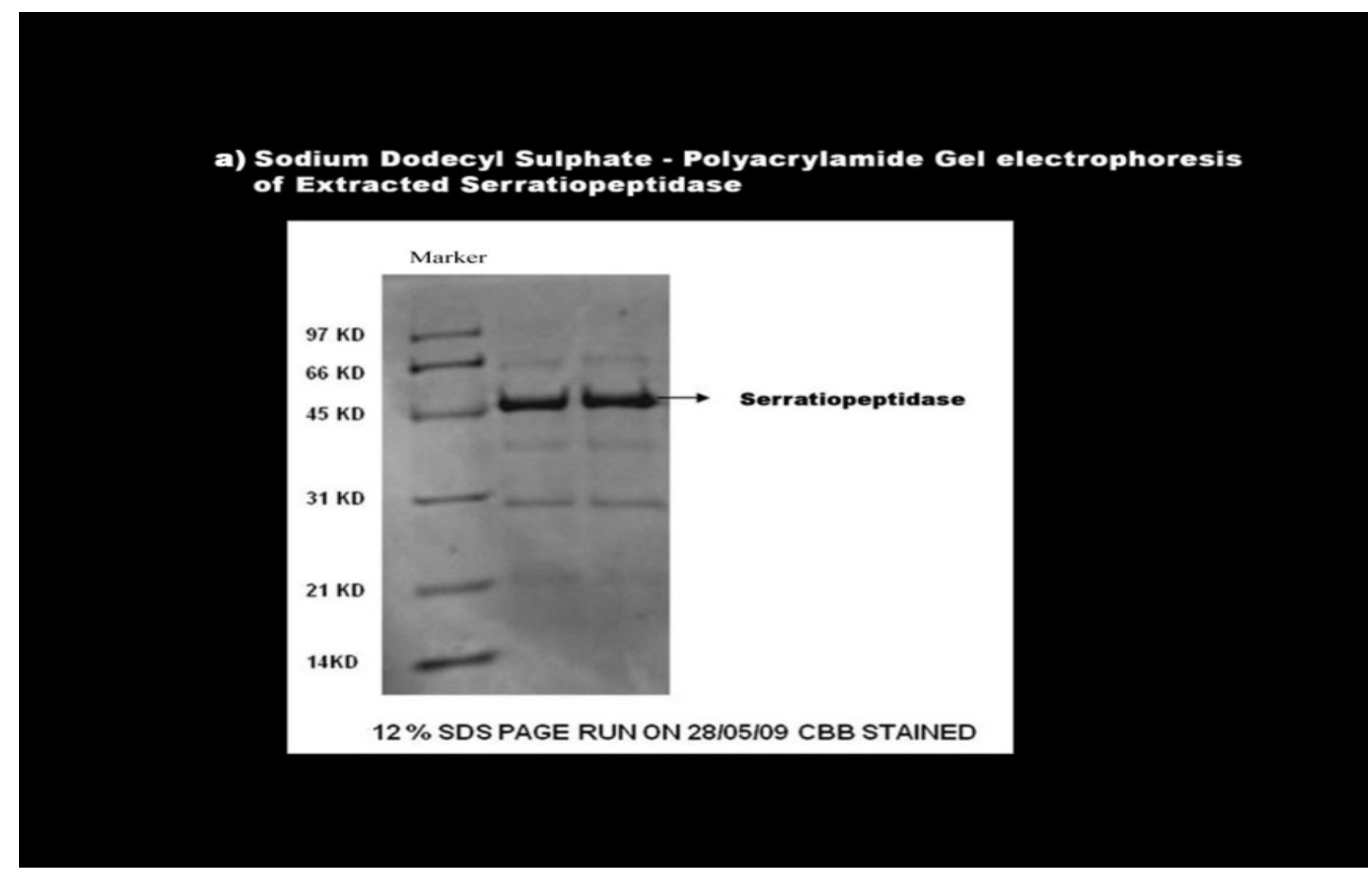

Plate C.

\subsection{Peptide Mass Fingerprinting}

A mass spectrum of the peptide mixture resulting from the digestion of a protein by an enzyme provides a fingerprint of great specificity. So specific, that it is often possible to identify the protein from this information alone.

This method of identification is much more reliable than using fingerprints based on PAGE migration patterns or HPLC retention times. However, peptide mass fingerprinting is limited to the identification of proteins for which sequences are already known, it is not a method of structural elucidation.

\subsection{Mascot Protein View}

The peptide sequence of STP obtained matched to PRZN_SERSP of NCBI database with a score of 530. The score 530 indicates high probability match and hence the enzyme can be 
confirmed as serratiopeptidase. The mass of the protein was determined to be 52203 Daltons. The enzyme also aids the taxonomical identification of Serratia marcescens. The protein sequence is stored in the database of matrix science as a database file (ID MSDB 20060831). This database file, reviews show 3239079 sequence and 1079594700 residues.

Match to: PRZN_SERSP Score: 530

Serralysin precursor (EC 3.4.24.40) (Extracellular metalloproteinase) (Zinc proteinase) (Serrapeptase) (Serrapeptidase) (Serratiopeptidase).-Serratia sp. (strain E-15) (Serratia marcescens (ATCC 21074)).

Found in search of J:IDATA_DELIVERYLCCMS FILES

JUNE2009\AGI\TIRUCHENGODE-HARI KRISHNA RAJ\A-PROTEIN.mgf

Nominal mass $\left(\mathrm{M}_{\mathrm{r}}\right)$ : 52203; Calculated $\mathrm{pI}$ value: 4.64

NCBI BLAST search of PRZN_SERSP against $\mathrm{nr}$

Unformatted sequence string for pasting into other applications

Taxonomy: Serratia sp. E-15

Fixed modifications: Carbamidomethyl (C)

Variable modifications: Oxidation (M)

Cleavage by Trypsin: cuts $\mathrm{C}$-term side of $\mathrm{KR}$ unless next residue is $\mathrm{P}$

Sequence Coverage: $\mathbf{2 7 \%}$

Matched peptides shown in Bold Red

1 MQSTKKAIEI TESNFAAATT GYDAVDDLLH YHERGNGIQI NGKDSFSNEQ

51 AGLFITRENQ TWNGYKVFGQ PVKLTFSFPD YKFSSTNVAG DTGLSKFSAE

101 QQQQAKLSLQ SWADVANITF TEVAAGQKAN ITFGNYSQDR PGHYDYGTQA

151 YAFLPNTIWQ GQDLGGQTWY NVNQSNVKHP ATEDYGRQTF THEIGHALGL

201 SHPGDYNAGE GNPTYRDVTY AEDTRQFSLM SYWSETNTGG DNGGHYAAAP

251 LLDDIAAIQH LYGANLSTRT GDTVYGFNSN TGRDFLSTTS NSQKVIFAAW

301 DAGGNDTFDF SGYTANQRIN LNEKSFSDVG GLKGNVSIAA GVTIENAIGG

351 SGNDVIVGNA ANNVLKGGAG NDVLFGGGGA DELWGGAGKD IFVFSAASDS

401 APGASDWIRD FQKGIDKIDL SFFNKEAQSS DFIHFVDHFS GAAGEALLSY

451 NASNNVTDLS VNIGGHQAPD FLVKIVGQVD VATDFIV 
Start - End Observed Mr(expt) Mr(calc) Delta Miss Sequence

$\begin{array}{llllll}44-57 & 792.9500 & 1583.8854 & 1583.7580 & 0.1275 & 0\end{array}$

K.DSFSNEQAGLFITR.E (Ions score 95)

$\begin{array}{lllllll}74-82 & 559.3500 & 1116.6854 & 1116.5492 & 0.1363 & 0 & \text { K.LTFSFPDYK.F }\end{array}$

(Ions score 33)

$\begin{array}{llllll}83-96 & 692.4000 & 1382.7854 & 1382.6678 & 0.1177 & 0\end{array}$

K.FSSTNVAGDTGLSK.F (Ions score 65)

$\begin{array}{lllllll}9-106 & 582.8300 & 1163.6454 & 1163.5571 & 0.0884 & 0 & \text { K.FSAEQQQQAK.L }\end{array}$

(Ions score 66)

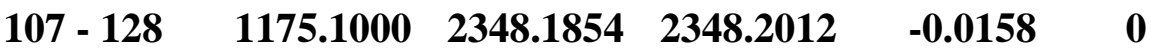

K.LSLQSWADVANITFTEVAAGQK.A (Ions score 109)

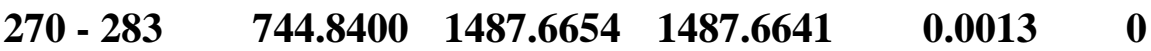

R.TGDTVYGFNSNTGR.D (Ions score 30)

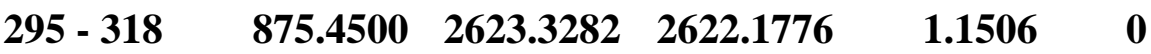

K.VIFAAWDAGGNDTFDFSGYTANQR.I (Ions score 55)

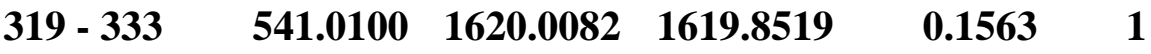

R.INLNEKSFSDVGGLK.G (Ions score 28)

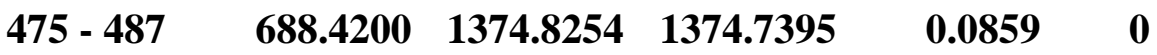

K.IVGQVDVATDFIV.- (Ions score 33)

$\begin{array}{llllll}475-487 & 688.4300 & 1374.8454 & 1374.7395 & 0.1059 & 0\end{array}$

K.IVGQVDVATDFIV.- (Ions score 49)

\section{>P1;PRZN_SERSP}

Serralysin precursor (EC 3.4.24.40) (Extracellular metalloproteinase) (Zinc proteinase)

(Serrapeptase) (Serrapeptidase) (Serratiopeptidase).-Serratia sp. (strain E-15) (Serratia marcescens (ATCC 21074)).

C;Species PRZN_SERSP: Serratia sp. (strain E-15) (Serratia marcescens (ATCC 21074)).

C;Family: Bacteria; Proteobacteria; Gammaproteobacteria; Enterobacteriales;

Enterobacteriaceae; Serratia. 
C;Accession: P07268;

C;EMBL; X04127; CAA27738.1; ALT_FRAME; Genomic_DNA.

\section{C;SRCDB SWISSPROT}

\subsection{Protein Hits}

The sequence when subject to matrix science protein hit, the enzyme method. Serralysin precursor protein (EC 3,4,24,140) (Extracellular metalloproteinase) (Zinc proteinase) (serratiopeptidase) from Serratia sp (strain E-15), Serratia marcescens (ATCC 21074) with a score of 530. Hence the protein is more similar to serratiopeptidase.

Protein hits $\quad$ : PRZN SERS Serralysin precursor (Ec 3.4.24.40) (Extracellular

Metalloproteinase) (Zinc proteinase) (Serrapeptase)

(Serrapeptidase) (Serratiopeptidase).-Serratia $s p$.

(Strain E-15) (Serratia marcescens (ATCC 21074)).

AB2633 conserved hypothetical protein Atu0461

[imported]-Agrobacterium tumefaciens (Strain

C58, Dupont)

Q3N3U3 9DELT Rubrerythrin.-Syntrophobacter fumaroxidans

MPOB.

AB2166 hypothetical protein alr2881 [imported]-Nostoc $s p$.

(Strain PCC 7120)

Q8EMU2 OCEIH Hypothetical protein OB2748.-Oceanobacillus iheyensis.

The enzyme also matched to some other proteins such as Atu 0461 from Agrobacterium tumefaciens with a score of 39, Rubrerythrin from Syntrophobacter fumaroxidans (score 37), also 2881 (inparted)-Nostoc sp (score 36), OB 2748 from Oceanbacillus iheyensis (Score 35), and also to some other un assigned proteins. The protein hits helps to identify the role of STP as enzymes that matched the sequence. Hence multifunctional activity of the STP enzyme is exposed.

\section{- Mascot Search Results}

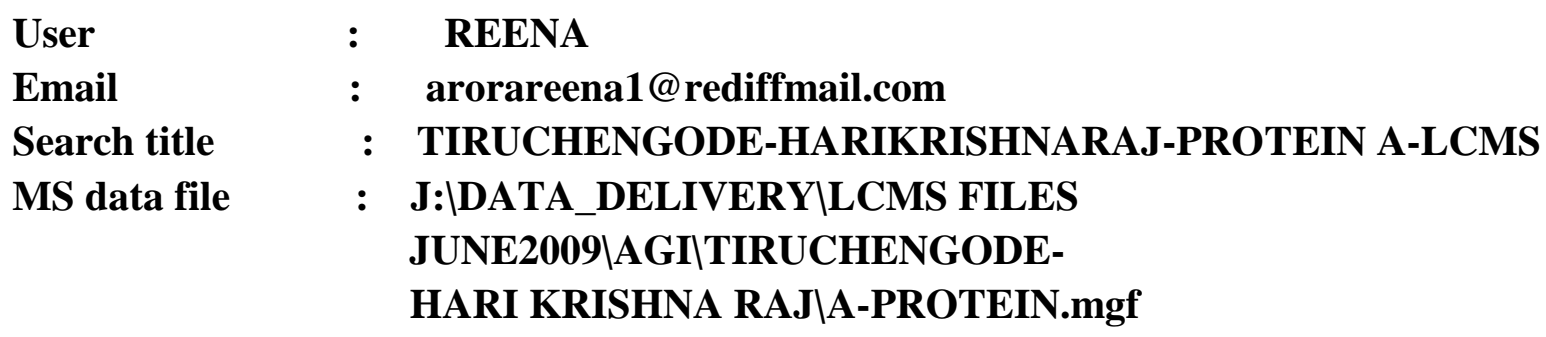




\section{Database}

Taxonomy

Timestamp
: MSDB 20060831 (3239079 sequences; 1079594700 residues)

: Bacteria (Eubacteria) (1478092 sequences)

: 11 Jun 2009 at 06:12:10 GMT

\section{Discussion}

This paper is to describe the identification of Serratia marcescens by 16s RNA sequence analysis and indirect approach towards the identification of the organism by the serratiopeptidase enzyme production and its characterization by peptide sequencing.

The red pigmented pure culture isolated from clinical specimen was given to the Helini Biomolecules Research institute, for the 16s r RNA sequencing analysis. This was done for the genetic identification of isolated pure culture. The DNA was isolated from the given culture and amplified by polymerase chain reaction with the help of forward and reverse primer. Then the amplified DNA was sequenced and the sequence was presented in the graphical from (chromatogram) which is shown in the figure1.0. The obtained sequences were compared to the closely related sequences of other bacteria and it was presented in the form of neighbour tree and phylogenetic construct (dendogram). These types of sequence analysis, studied by other researchers were not represented elaborately in their research, as described in this paper. Hence there is little or no scope for a comparative discussion, in this way this work represents a novel approach towards genetic characterization and identification of an organism.

Various investigations have reported the isolation and characterization of Serratia proteases (Liu, 1961; Maeda, 1979; Miyata et al,. 1970). In this study, the isolation of pure culture from the clinical specimen was identified for the production of serratiopeptidases. This enzyme was purified by ammonium sulfate precipitation, DEAE cellulose column chromatography. (Aiyappa and Harris, 1976; Lyerly and Kreger, 1979; Decedue et al,. 1979; Miyata et al,. 1970). After purification the enzyme was subject to SDS-PAGE analysis and peptide mass finger printing.

An enzyme of low specificity, which digests proteins to a mixture of free amino acids and diand tri- peptides, is not a good choice. So the choice of enzyme for MALDI-TOF analysis was very important. A complex mixture containing large numbers of components of similar mass will result in a lot of overlapping peaks. A further consideration for MALDI analysis is that the low mass region, below $\sim 500 \mathrm{Da}$, is obscured by the presence of matrix peaks.In general, it is best to use enzymes of specificity equal to or greater than trypsin.

Setting the number of allowed missed cleavage sites to zero simulates a limit digest. If the digest is perfect, with no partial fragments present, it will give maximum discrimination and the highest score. If the digest mixtures include some partials that is, peptides with missed cleavage sites, a setting of 1 , or may be 2 is chosen for missed cleavage sites. With all these considerations the peptide sequencing was performed to the fullest possibility and was very accurate in identifying serratiopeptidase and subsequently Serratia marcescens. Thus a complete indirect and direct genetic identification of Serratia marcescens was achieved. 


\section{Acknowledgement}

We are grateful to the management of Sengunthar Arts and Science college, Tiruchengode, India, for providing the necessary facilities to carry out this research.

\section{References}

Aiyappa, P. S., \& J. O. Harris. (1976). The extracellular metalloprotease of Serratia marcescens its purification and characterization. Mol.cell. Biochem., 13, 95-100.

Conroy, M. C., N. H. Bander \& I. H. Lapow. (1975). Effects in the rat of intradermal injection of purified proteinases from Streptococcus and Serratia marcescens. Proc. Soc. Exp. Biol. Med., 159, 801-806.

Decedue, C., E.Broussard, A. Larson \& H. Braymer. (1979). Purification and Characterization of the extracellular proteinase of Serratia marcescens. Biochem. Biophy. Acta., 569, 293-301.

Dorwart, B., B. E. Abrutya \& H. R. Schumacher. (1975). Serratia arthritis: Medical eradication of infection in a patient with rheumatoid arthritis. J. Am. Med. Assoc., 225, 1642-1643.

Graber, C. D., L. S. Higgins \& J. S. Davis. (1965). Seldom-encountered agents of bacterial meningitidis. J. Am. Med. Assoc., 192, 114-118.

Liu, P. V. (1961). Observations on the specificities of extracellular antigens of the genera Aeromonas and Serratia. J. Gen. Microbial., 24, 145-154.

Lyerly, D., \& A. Kreger. (1979). Purification and Characterization of Serratia marcescens metalloprotease. Infect. Immun., 24, 411-421.

Maeda, H. (1979). Array of proteolytic enzymes by fluorescence polarization. Anal. Biochem., 92, 222-227.

Miyata, K., K. Maejima, K. Tomoda \& M. Isono. (1970). Serratia protease.Part I. Purification and general properties of the enzyme. Agri. Biol. Chem., 34, 310-318. www.matrixscience.com

\section{Copyright Disclaimer}

Copyright reserved by the author(s).

This article is an open-access article distributed under the terms and conditions of the Creative Commons Attribution license (http://creativecommons.org/licenses/by/3.0/). 